\title{
LA INTERACCIÓN FAMILIAR EN UN AMBIENTE SALUDABLE ${ }^{1}$
}

\author{
Ana Teresa López de Llergo Villagómez \\ Luz María Cruz de Galindo
}

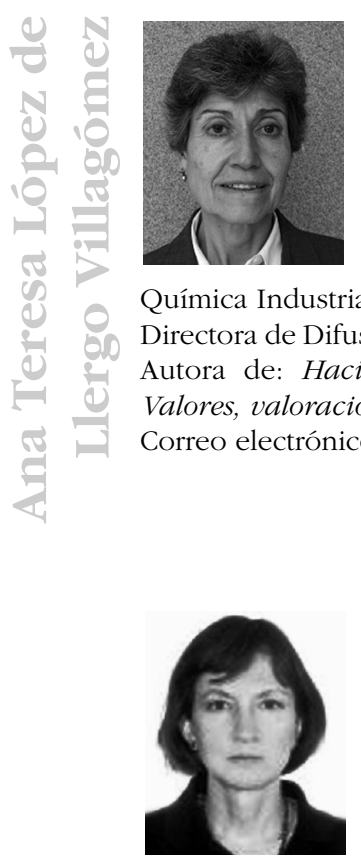

Doctora en Ciencias de la Educación, Universidad de Navarra

Maestra en Pedagogía, Universidad Panamericana.

Licenciada en Ingeniería Química y Química Industrial, Universidad Iberoamericana.

Directora de Difusión Cultural, Universidad Panamericana. Autora de: Hacia un desarrollo humano, Ed. Limusa; Valores, valoraciones y virtudes, Ed. CECSA.

Correo electrónico: [alopezde@mx.up.mx].

Maestra en Educación Familiar, Universidad Panamericana.

Licenciada en Trabajo Social, UNAM.

Maestrante en Historia del Pensamiento, Universidad Panamericana.

Maestrante en Trabajo Social, Universidad Autónoma de México.

Docente del EDAC, IEF, Escuela para Padres del Centro Escolar Cedros y Enlace.

Correo electronico: [lucy@olinala.com].

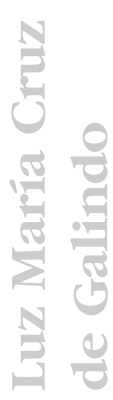

Ponencia para el XII Congreso Internacional Ciencia y Vida. Cuarto Claustro General de ULIA, Valencia (España) 2006, 1, 3 y 4 de julio. 


\title{
RESUMEN
}

A partir de una radiografía del estado general de la juventud y de dos datos que arrojan: la gran desconfianza a lo relacionado con la política, y la confianza en la institución familiar, en este trabajo se reflexiona sobre algunas pautas indispensables en esta institución para minimizar la brecha entre lo privado y lo público, y para fortalecer el capital humano. Se indican políticas sociales para respaldar la ardua e importante tarea de la familia. Finalmente, se sugiere una serie de recomendaciones cuya base implica la fidelidad entre todos los miembros de cada dinastía.

Palabras clave: interacción; familia; políticas sociales; ambiente saludable; esferas.

\begin{abstract}
From an X-ray of the youth general status and some data that these throw: the great distrust related to politics, and the trust in the family institution, in this job you think about some indispensable guidelines in this institution to minimize the gap between private and public and to strengthen the human capital. Social politics are indicated to endorse the harsh and important family task. Finally, a series of recommendations are given, which basis imply the fidelity between all the members of each dynasty.
\end{abstract}

\section{Key words :}




\section{INTRODUCCIÓN}

Los resultados preliminares de la Segunda Encuesta Nacional de Juventud 2005 (ENJ) que se llevó a cabo en la República Mexicana, publicados en la página del Instituto Mexicano de la Juventud (IMJ) ${ }^{2}$, el 22 de mayo de 2006, tienen como finalidad el diseño de políticas públicas más acordes con nuestra realidad. En el estudio - elaborado a nivel nacional y en el cual se entrevistaron a 12,815 jóvenes-, el IMJ detectó que 44\% de ellos no tiene interés en la política; $22 \%$ afirma que una de las razones para ese desinterés es "porque los políticos no son honestos".

La investigación reveló que los jóvenes que estudian ascienden a $43.7 \%$, en tanto quienes trabajan son $28.8 \%$, y $22.1 \%$ de los jóvenes no estudian ni trabajan. Sobre la edad en que tuvieron su primera relación sexual, la ENJ/2005 indica que el promedio nacional es de 17.5 años; siendo para el caso de los hombres los 17 años, en tanto que para las mujeres los 18. Esta radiografía nacional de los jóvenes mexicanos muestra que más de 90\% tiene conocimiento de los métodos anticonceptivos, y es el condón (86.9\%) el más utilizado entre quienes emplean estos métodos.

Sobre las expectativas de su vida, las mujeres jóvenes desean preferentemente formar una familia y tener hijos (48.4\%), mientras que los hombres jóvenes ansían obtener un buen trabajo (54.4\%). En relación a su participación en asociaciones, la mayoría de los jóvenes lo hacen en deportivas (38.4\%), estudiantiles (13.1\%) y religiosas (13.1\%).

2 Cfr. [www.imjuventud.gob.mx]. 
La ENJ/2005 pidió a los jóvenes que calificaran de 1 a 10 en qué institución confían más. Los resultados en promedio fueron: la familia (con una calificación de 9.1), los médicos (8.5) y la escuela (8.3). Las instituciones con menos calificación fueron: la policía (5.9), los diputados federales (6) y los partidos políticos (6). Los resultados finales de la ENJ, elaborada por el Centro de Investigación y Estudios sobre Juventud del Instituto Mexicano de la Juventud, se dieron a conocer en octubre de 2006.

Por lo pronto, vale la pena reflexionar en dos actitudes: la gran desconfianza hacia todo lo relacionado con la política, y la confianza en la institución familiar. Esto genera una brecha entre lo privado y lo público, lo individual y social, respectivamente. Además, es una lástima que los propios jóvenes - con su iniciación tan temprana a la vida sexual, muchas veces fuera del matrimonio, y la ruptura entre el significado unitivo y procreativo de la sexualidad- estén contribuyendo de manera, tal vez inconsciente, al empobrecimiento del capital humano y la desvirtualización de las tareas fundamentales de la familia. Con esta tónica es difícil lograr el legítimo anhelo de la permanencia de la auténtica familia y, paradójicamente, se propician los resultados contrarios a los deseados, debido a las conductas irreflexivas y muchas veces manipuladas por intereses hedonistas y mercantilistas, y a un entramado social que se ha debilitado.

Pero es necesario indagar las características del grupo familiar en el que han depositado su confianza; pues tal vez los jóvenes ya han experimentado la ruptura entre sus progenitores y la presencia de nuevas uniones. Todas estas relaciones sociales provocan un desdibujamiento del verdadero 
rostro de la institución familiar. Sin duda, las rupturas van acompañadas de rencores, faltas de respeto a los derechos de los esposos y de los hijos, y la intrusión de personas que pueden considerarse como advenedizas; o la triste experiencia de encontrar que quien suplanta al padre o a la madre es mejor que el propio, así como el miedo de perderlo más adelante. Lo que para generaciones pasadas era tal vez común (matrimonios integrados, familias unidas capaces de superar las diferencias), ahora se está convirtiendo en la práctica ensayoerror que deja heridas profundas.

\section{LAS POLÍTICAS SOCIALES COMO RESPALDO A LA FAMILIA}

Las políticas sociales se refieren a la manera como interviene el Estado para resolver los problemas que aquejan a los grupos más vulnerables de la sociedad. La finalidad es implementar programas de acción en materia social para realizar ajustes al interior de las estructuras, que moralicen la economía liberal y eviten, en lo posible, las desigualdades sociales injustas.

Esping-Andersen en su obra, Los tres mundos del Estado de bienestar ${ }^{3}$, presenta cuatro esferas de la estructura social: mercantil, estatal, doméstico-familiar y relacional. Cualquiera de ellas puede proveer de bienestar social a la población, y hacerlo simultáneamente. Las esferas son un complejo de instituciones

Cfr. ADELANTADO, José; NOGUERA, José A.; RAMBLA, Javier; SÁEZ, Luis. "Las relaciones entre estructura y políticas sociales: una propuesta teórica", en ARTEAGA Basurto, Carlos; SOLÍS San Vicente, Silvia (coordinadores). La política social en la transición. ENTS, UNAM y Plaza y Valdés, Editores. México 2001, pp. 51 a 75 . 
y mecanismos de coordinación de la acción social, o dimensiones de esa acción; están cruzadas transversalmente por individuos y grupos y por los diversos ejes de desigualdad.

La esfera mercantil se refiere a la producción e intercambio material; se relaciona con la provisión que realizan las empresas privadas en educación, salud y vivienda. En esta esfera se jerarquizan las relaciones sociales según la capacidad de vender o comprar mercancías. Así surge un proceso de mercantilización ${ }^{4}$ de las relaciones sociales que es clave para entender la política social 5 . No profundizaremos más en ella, porque no es la que nos interesa para nuestra disertación.

La esfera doméstico-familiar y de parentesco abarca las actividades realizadas dentro de las unidades mínimas de coresidencia en las que se ejecuta una forma de trabajo, que varios procesos históricos y sociales han atribuido a las mujeres. Este trabajo consiste en la prestación de cuidados personales (trabajo reproductivo) al margen de cualquier remuneración monetaria, y se concreta en actividades muy variadas: cocinar, lavar, mediar entre el hogar y el mercado (consumo), o entre el hogar y el Estado (uso de los servicios públicos). En un sentido amplio, la esfera doméstico-familiar trasciende la localización física o espacial del locus donde se desarrolla el trabajo doméstico y el cuidado familiar, y llega a la dimensión de parentesco que (re)introduce nuevas relaciones como las que se establecen entre personas con vínculos consanguíneos

Cuando una relación social se extrae del circuito mercantil, se produce la desmercantilización.

La política social se articula en un conjunto de decisiones y actuaciones público-administrativas, generadas en la esfera estatal, que inciden directamente y de formas diversas sobre la organización y distribución de los recursos de bienestar, y lo hace mediante regulaciones, servicios y transferencias. 
y legales, en tanto se realicen tareas de cuidado, mantenimiento y reproducción de la vida de los individuos. La importancia de estos vínculos intergeneracionales, que se articulan en función del principio económico de la reciprocidad — diferente de la solidaridad altruista, o el intercambio de bienes entre iguales, que descansa sobre un sentimiento de obligación socialmente construido, sumamente vinculante- se refleja en muchas circunstancias: abuelos que van a buscar a sus nietos al colegio, hijas y nueras que cuidan de padres y suegros que no viven bajo el mismo techo... La reactivación de los lazos de parentesco no puede extenderse exclusivamente por variables culturales, responde también a los impactos de las políticas sociales; así, en un contexto de privatización y desregulación del mercado de trabajo, se combate la incertidumbre estrechando los lazos familiares.

La esfera estatal incluye el aparato institucional y administrativo público, y el conjunto de las actuaciones generadas en ese mismo marco. Desde su posición central en una realidad social, irradia a todos los ámbitos sus medidas de definición, reproducción y cambio de la estructura social (de la cual ella misma es parte integrante). Es fundamental en la organización de la desigualdad social, en asuntos distributivos (impuestos, legislación económica, presupuestos, políticas públicas) y en la reproducción simbólica de las jerarquías sociales. Esta esfera, y en concreto la política social, puede tener gran importancia de cara a la modulación y constitución de algunas divisorias sociales que no pasan necesariamente por el mercado ni por la esfera doméstica (ciudadanía, clientes de las burocracias públicas, empleados/beneficiarios de los servicios sociales, etcétera). Por eso, su acción puede estar condicionada por su mayor o menor permeabilidad a las presiones 
de los grupos organizados que se forman en la esfera relacional. Así, uno de sus instrumentos será la puesta en marcha de los distintos tipos de políticas sociales. Obviamente, las políticas sociales son diseñadas por la esfera estatal, pero ello no impide que su formación y contenidos puedan verse, y de hecho se vean, notoriamente condicionados por la estructura y la dinámica de las demás esferas.

La esfera relacional, compuesta por las acciones sociales supra-individuales, canaliza intereses y necesidades (no sólo materiales) de las personas, mediante distintos grupos distribuidos en asociaciones formales y en grupos comunitarios (informales). No se identifica con la esfera mercantil, pues en la relacional se consideran las orientaciones de valor de los individuos, e incluye vínculos y actividades claramente públicas. Tampoco puede reducirse a una interpretación "discursiva" de la "esfera pública", pues en la esfera relacional no sólo se discute o se forma opinión, también se organizan solidaridades y grupos de cara a la acción colectiva. En la actividad relacional, además de la comunicación en sí misma, surgen intereses económicos, políticos y otros, que pueden originar relaciones de dominación y desigualdades de poder y recursos.

Dentro de la esfera relacional podemos distinguir:

- La subesfera asociativa o pública: Comprende asociaciones más o menos institucionalizadas, y los movimientos sociales (diversidad de formas organizativas alrededor del género, edad, etnia, pacifismo, ecologismo, etcétera). Es la llamada "sociedad civil", donde los agentes no son completamente autónomos ni espontáneos. La idea de subesfera pública resalta el carácter conflictivo de este ámbito y las capacidades desiguales de organización de los agentes, de acuerdo 
con su posición social. La capacidad de organización de esta subesfera puede ser un eje de desigualdad importante en la configuración de políticas sociales. Su importancia es capital en la mediación de intereses colectivos, la formación de la opinión pública y el ejercicio de los derechos de la ciudadanía. La idea de subesfera asociativa evidencia las actuaciones como filtro de los intereses y de las aspiraciones de los agentes sociales, pues canaliza sus demandas y delimita los contornos de las acciones colectivas y de las presiones de los distintos agentes sobre las demás esferas.

- La subesfera comunitaria: Su núcleo es el sentimiento de pertenencia o vínculo con una comunidad. En su seno, los distintos agentes instrumentan estrategias y actúan en función de sus intereses, o bien sus relaciones deben entenderse como de reciprocidad, y no tanto de agrupación de intereses y opiniones similares, o solidaridades de grupo. El referente común de los agentes es la propia comunidad, en la cual se opera a un nivel informal, a diferencia de la relativa formalidad institucional o semi-institucional que caracteriza a la subesfera asociativa (centrada más en la consecución de espacios institucionales y de influencia respecto de otras esferas, como la estatal o la mercantil). En esta subesfera, las acciones suelen derivar en un conglomerado de redes de reciprocidad, ligadas a vínculos intracomunitarios de muy diverso signo - vecinales, de amistad, etcétera- y que pueden proveer también cuidados o prestaciones relevantes para estudiar la política social. Al igual que ocurriría con la esfera doméstica, una subesfera comunitaria fuerte y articulada puede evitar situaciones de ruptura social por ausencia de políticas de bienestar público (puede sustituir parcialmente la redistribución estatal por reciprocidad comunitaria). Ello no implica que en esas redes de reciprocidad no 
puedan existir también asimetrías y relaciones de desigualdad (en cuanto a capacidad de influencia o disponibilidad de recursos comunitarios).

Los actores colectivos manifiestan la estructura social en el terreno de la acción pero, a su vez, pueden reconfigurar esa estructura vinculándose de variadas formas a esferas específicas. Las interrelaciones entre la estructura social y la política social son recursivas: esta última no sólo se genera a partir de la primera, también contribuye a los procesos de estructuración de las sociedades y provoca cambios o permanencias - de manera directa o indirecta- en una esfera o en varias; por ejemplo, puede tener un impacto en la asociativa que, a su vez, afecte a la estatal.

La política social es un dispositivo gubernamental que permite la gestión de la desigualdad y asigna a cada esfera de la estructura -mercantil, estatal, doméstica y relacional- un papel en la satisfacción de las necesidades, reequilibrando el flujo de relaciones entre ellas de forma continua. Esta concepción de estructura social con base en las cuatro esferas se conecta con los estudios sobre la división social del bienestar. Una relación social puede trasvasarse de sector a sector, o de un sector a varios sectores. Los flujos no necesitan ser bidireccionales, pueden ser pluridireccionales. Según EspingAndersen, cualquier potencial transformador y subversivo de la política social radica en su capacidad de desmercantilización.

\section{LA INTERACCIÓN FAMILIAR}

La familia, según la definición clásica de Pedro-Juan Viladrich, es el hábitat natural para nacer, crecer y morir con 
toda dignidad de un ser personal ${ }^{6}$. Matrimonio y familia —afirma Benedicto XVI 7- no son una construcción sociológica casual, fruto de situaciones particulares históricas y económicas. Por el contrario, la relación entre el hombre y la mujer hunde sus raíces en la esencia más profunda de la persona. No puede separarse de la pregunta siempre antigua y siempre nueva sobre uno mismo: ¿quién soy?, del lazo indisoluble entre espíritu y cuerpo. El ser humano es, de hecho, alma que se expresa en el cuerpo, y cuerpo que es vivificado por un espíritu inmortal; su cuerpo no es simplemente cuerpo, y lo biológico no es sólo biológico, sino expresión y cumplimiento de la propia humanidad.

Ninguno de nosotros se pertenece exclusivamente a sí mismo; cada uno está llamado a asumir en lo más íntimo de sí, su propia responsabilidad pública. El matrimonio, como institución, no es por tanto una injerencia indebida de la sociedad o de la autoridad, una imposición desde el exterior en la realidad más privada de la vida, sino una exigencia intrínseca del pacto de amor conyugal y de la profundidad de la persona humana. Por ello, la familia es también el entorno más adecuado ${ }^{8}$ para aprender las normas morales y las pautas de conducta que rigen todo tipo de convivencia. Esa centralidad sigue vigente. De hecho, como lo reflejó la ENJ, la importancia de la familia para los jóvenes — pero no en exclusiva

Cfr. Agonía del matrimonio legal, Instituto de Ciencias para la Familia, Ediciones Universidad de Navarra S.A., 2a edición, Pamplona 1989, p. 196. Cfr. Discurso en la apertura del Congreso Eclesial de la Diócesis de Roma sobre "Familia y comunidad cristiana: formación de la persona y transmisión de la fe" en la Basílica de San Juan de Letrán, el lunes 6 de junio de 2005.

8 LECHNER, Norbert. Cultura juvenil y desarrollo humano. JOVENes, Revista de Estudios sobre Juventud Edición: año 8, núm. 20.México, DF, enero-junio 2004. pp. 12-27. 
para ellos- es elevada, tal vez porque el entramado social se ha vuelto complicado, cada filamento está retorcido y la tensión lo rompe.

En la medida en que aumentan las dificultades de crear vínculos sociales y referentes colectivos, las personas tienden a cerrarse en grupos más pequeños. En algunos casos, las personas se retiran a la vida familiar porque creen poder prescindir de la sociedad. El encierro en ciertos condominios ilustra la retirada de las elites. En muchos otros casos, cuando la realidad social es vivida como algo ajeno y hostil, la familia se vuelve el último refugio del yo. Puede ser que el repliegue a lo privado abarque, además de la familia, a los amigos.

De ahí la importancia de conservar la verdadera identidad de la familia: sin dejar de ser un refugio seguro ha de ser también la mejor escuela de socialización. Para ello, el núcleo familiar debe tener apertura y también intimidad; estar preocupada por su entorno, con proyectos y planes; ser sensible a los problemas sociales, con interés por solucionarlos. Sólo una familia así podrá interactuar e influir en el ambiente sociocultural.

En la actualidad, al mismo tiempo que se confirma su rol central, la familia se encuentra sometida a un profundo cambio. Por una parte, surgen modificaciones drásticas en las relaciones hombre- mujer; el acceso de esta última al mundo laboral afianza su autonomía individual y le permite logros significativos, que inciden en los estilos de convivencia intra y extra familiar, con resultados no siempre deseables. Por la otra, también se flexibilizan los lazos entre padres e hijos; es notoria, por ejemplo, la dificultad de los padres para decir no y establecer límites a sus hijos. Existe un debilitamiento de la autoridad 
paterna como consecuencia de la privatización de la familia y su desacople de lo social. Los padres esperan en vano que la sociedad ratifique la figura histórica propia de los progenitores como representantes de la autoridad. A la inversa, la sociedad muchas veces ya no consigue apoyarse en la función paterna para lograr que sean descubiertos los valores y las normas que regulan la convivencia. Ambos fenómenos indican que la familia, como institución, se ha vuelto auto-referencial.

Si este proceso no se frena, la familia, como unidad grupal fundamental y natural ${ }^{9}$, dejará de ser el medio idóneo para lograr el desarrollo integral de sus miembros, y la sustentabilidad social, económica y del desarrollo cultural. Ya no servirá como plataforma que impulsa a cada persona al logro de sus fines existenciales, ni será capaz de enfrentar los distintos retos actuales en el contexto de la globalización. Entonces, fortalecer a la familia es una única oportunidad para dirigir los problemas sociales de manera holística; además, las familias fuertes y estables contribuyen al mantenimiento de una cultura de paz y promueven el diálogo entre civilizaciones y entre los diversos grupos étnicos.

\section{EL AMBIENTE SALUDABLE, CAUSA Y EFECTO DE LA ADECUADA INTERACCIÓN FAMILIAR}

En el mundo de hoy, en el que se difunden concepciones equívocas sobre el ser personal, la libertad, el amor humano...

Cfr. Declaración Universal de los Derechos Humanos, Art. 16. 
no podemos dejar de presentar la verdad sobre la familia ${ }^{10}$. Por desgracia, está creciendo el número de separaciones y divorcios, que rompen la unidad familiar y crean muchos problemas a los hijos, víctimas inocentes de estas situaciones. La estabilidad de la familia está en nuestros días particularmente en peligro; para salvaguardarla es necesario ir con frecuencia contra la corriente de la cultura dominante, y esto exige paciencia, esfuerzo, sacrificio y búsqueda incesante de la comprensión mutua. Pero también hoy les es posible a los cónyuges superar las dificultades y mantenerse fieles a su vocación. La unidad y la firmeza de las familias ayudan a la sociedad a respirar los auténticos valores humanos.

La interacción familiar adecuada implica una fidelidad entre todos sus integrantes, empezando por los cónyuges, el sí de cada uno - en el que se funda la familia - incluye la dimensión del tiempo; es un más allá del momento presente: en su totalidad, el sí significa siempre, constituye el espacio de la fidelidad. Sólo en este clima puede aumentar la confianza. De esta manera los hijos, fruto del amor, creerán en la humanidad aun en tiempos difíciles. La relación paterno-filial implica la libertad del otro que se traduce en un compromiso con la verdad y con el bien, para construir con los demás algo en común.

La familia debe enfrentar una serie de desafíos para cumplir las tareas que le son propias: ofrecer amor incondicional, educar para el trabajo y la convivencia, ser la primera escuela de socialización. Sólo sin desvirtuarse podrá ser una institución naria del Consejo Pontificio para la Familia. CIUDAD DEL VATICANO, domingo, 14 mayo 2006. 
social fundamental, decisiva e insustituible para el bien común de los pueblos; fundada en el matrimonio, se constituye en la célula vital y el pilar de la sociedad. Es una realidad a la que todos los Estados deben dedicar la máxima consideración.

Pero la esfera estatal no siempre ayuda a la domésticofamiliar, y es necesario, en primer lugar, establecer una relación intrafamiliar tan sólida que pueda mantenerla inmune a las influencias nocivas y, como por ósmosis, expandir su salud a la sociedad mediante el contagio a otras familias. Y, por otra parte, debe insistirse en que los gobiernos, las organizaciones internacionales y los miembros de la sociedad civil lleven a cabo acciones de protección a la familia, con programas para fortalecer la estabilidad de esta institución ${ }^{11}$. Pero, sobre todo, la misma familia debe de asumir su papel como comunidad de vida y amor. El camino idóneo será filtrar las influencias ambientales para regresarlas depuradas y transformar el entorno. La mejora de la sociedad empieza y concluye en la familia. La salud social —entendida como la gradación de los estados de felicidad colectiva o excelencia grupal en ámbitos sociales ${ }^{12}$ - encuentra su fundamento en la de cada una de las familias.

En este momento histórico hay que transformar en positivos, las tendencias y los desarrollos negativos, como el envilecimiento del amor que aplasta y oprime a los otros; la explotación irracional de la naturaleza, incluida la humana pues esto, más

${ }^{11}$ Cfr. Doha, 30 de noviembre de $2004, \mathbf{1 0}^{\circ}$ aniversario del Año Internacional de la Familia. En: [www.Dicf.org.ga/english/index.html]. Los eventos preparatorios para esta conferencia reúnen puntos de vista de mandatarios gubernamentales, académicos, grupos religiosos, ONGs y miembros de la sociedad civil.

${ }^{12}$ Cfr. PÉREZ Adán, José, La salud social, Editorial Trotta, Madrid 1999, p. 14. 
que liberar, destruye. Sin embargo, ningún hombre y ninguna mujer, con sus propias fuerzas, pueden encontrar y transmitir a sus hijos el amor y el sentido de la vida de cada cual; se requiere la ayuda de una autoridad y una credibilidad superiores: Dios, para los creyentes.

\section{CONSIDERACIONES FINALES}

Las cuatro esferas de las que hemos hablado han de interactuar para lograr un ambiente saludable, cada una debe apoyarse y complementarse con la actividad de las otras. No obstante, la esfera doméstico-familiar, por ser la más próxima a la persona humana, necesita ser privilegiada por las otras tres; sobre todo porque de ella depende que el resto de las esferas cumplan bien sus tareas. En la familia se gestan las personas que se integrarán al mundo del mercado, del Estado y de la sociedad civil. La esfera estatal, por sus recursos y prerrogativas, es la responsable de promover la existencia de un ámbito domésticofamiliar adecuado para el desarrollo de cada una de las personas que lo integran. Algunas de las acciones para lograrlo son:

- Fortalecer las políticas y programas que habiliten a la familia para romper con el ciclo de pobreza.

- Evaluar y revaluar las políticas de población gubernamentales particularmente en países de baja reposición en sus índices de natalidad.

- Ofrecer soporte a la familia en los cuidados a las personas mayores y a las personas con discapacidad.

- Apoyarla en los tratamientos del VIH sida y otras pandemias, incluyendo malaria y tuberculosis.

- $\quad$ Elegir medidas efectivas para ayudarla en tiempos de paz y de guerra. 
- Proteger los derechos del matrimonio y la familia.

- Hacer efectivas la cooperación e igualdad entre los cónyuges, y la relación marital comprometida y duradera.

- Establecer políticas y prácticas adecuadas para condenar y remediar situaciones de abuso dentro de las relaciones matrimoniales y familiares, incluyendo el establecimiento de agencias públicas que otorguen asistencia a hombres, mujeres, niños y, en general, familias en crisis.

- Favorecer la igualdad de oportunidades políticas, económicas y sociales para las mujeres y los varones.

- Animar a que padres y madres se involucren en la educación de sus hijos. Ambos tienen el primordial derecho de elegir el tipo de educación para sus hijos.

Pero la esfera doméstico-familiar no es un mero ente receptivo, sus integrantes deben esforzarse en:

- Lograr que el trabajo fuera del hogar, tanto del hombre como de la mujer, tengan una repercusión efectiva en la esfera mercantil y en la estatal.

- Procurar un equilibrio de roles dentro de la familia que irradie paz al ambiente social.

- Facilitar expresiones de afecto y apoyo dentro de la familia que proporcionen a sus miembros una seguridad que más adelante se reflejará en sujetos maduros.

Las personas integran en su vida cotidiana las actividades típicas de cada esfera, las cuales, como se ha dicho, no son excluyentes, porque la vida humana es una unidad que se expresa en diferentes ámbitos y circunstancias. Lograr la armonía de las distintas actividades requiere de reflexión y prudencia. 


\section{REFERENCIAS BIBLIOGRÁFICAS}

ARTEAGA Basurto, Carlos; SOLÍS San Vicente, Silvia (coordinadores), La política social en la transición., ENTS, UNAM y Plaza y Valdés, editores., México., 2001.

BENEDICTO XVI, Los desafíos de la familia., Discurso a la asamblea plenaria del Consejo Pontificio para la Familia., Ciudad del Vaticano., mayo 14., 2006.

LECHNER, Norbert., "Cultura juvenil y desarrollo humano"., JOVENes, Revista de Estudios sobre Juventud., año 8., núm. 20., México., enero-junio., 2004.

PÉREZ ADÁN, José., La salud social., Editorial Trotta., Madrid., 1999.

VILADRICH, Pedro-Juan., Agonía del matrimonio legal., Instituto de Ciencias para la Familia., Ediciones Universidad de Navarra S.A., 2o edición., Pamplona., 1989.

DECLARACIÓN UNIVERSAL DE LOS DERECHOS HUMANOS., artículo 16.

Discurso en la apertura del Congreso Eclesial de la Diócesis de Roma sobre Familia y comunidad cristiana: formación de la persona y transmisión de la fe., Basílica de San Juan de Letrán., junio 6., 2005. En: [www. Dicf.org.ga/english/index.html].

Los eventos preparatorios para esta conferencia reúnen puntos de vista de mandatarios gubernamentales, académicos, grupos religiosos, ONGs y miembros de la sociedad civil. En: [www.imjuventud.gob.mx]. 